children presented with hemiparesis and one with facial paresis, hypotonia and gait impairment. Neuroimaging with MRI, MRA, and/or CT showed occlusion or stenosis of the middle cerebral artery in 3 cases or nucleo capsular signal alteration without vessel occlusion in one. The incubation time from rash to onset of stroke was 2 to 30 days. Congenital prothrombotic abnormalities present in 2 cases, and a concurrent streptococcal bacteremia in one other may have contributed to the cerebrovascular disease. Treatment included acyclovir and acetylsalicylic acid (ASA). One patient developed cerebral hemorrhage and ASA was discontinued. The 3 patients with hemiparesis had persistent deficits on follow-up. The facial palsy resolved. (Losurdo G, Giacchino R, Castagnola E, et al. Cerebrovascular disease and varicella in children. Brain Dev July 2006;28:366-370). (Respond: Dr Raffaella Giacchino, Infectious Diseases Unit, G Gaslini Children's Hospital, Largo G Gaslini 5, 16147 Genoa, Italy).

COMMENT. Pathogenic mechanisms for varicella-associated stroke include vasculitis, acquired protein S deficiency, antiphospholipid and anticardiolipin antibodies, and lupus anticoagulant activity. No common mechanism is described. Antiphospholipid antibody was positive in 2 of the above 4 cases; it became negative after 1 month follow-up. VZV infection is a rare but significant cause of stroke in children.

Varicella and delayed stroke. Acute hemiplegia developed 7 weeks to 4 months after varicella infection in 4 children in Japan (Ichiyama T et al. Pediatr Neurol 1990;6:279281). Carotid angiography showed segmental narrowing and occlusion of the middle cerebral artery, and cerebral angiitis was cited as the cause. The frequency of delayed stroke was estimated at 1 in 6500 cases of varicella in Japan.

\title{
VESTIBULAR NEURITIS AND ADENOVIRUS
}

A 4-year-old boy with vestibular neuritis and a serological diagnosis of adenovirus infection is reported from the University of Siena, Italy. He was admitted with paroxysmal vertigo, vomiting and unsteadiness, leaning left on attempted walking or during the Romberg test. A similar episode had occurred one year before; symptoms lasted 1 week and the patient was not hospitalized. On present examination, spontaneous horizontal nystagmus to the right decreased with visual fixation. EEG and ENT examinations were normal, and showed no evidence of seizure disorder or middle ear disease. Family history was negative for migraine. On partial recovery within a few days, a water caloric test showed no response in the left ear. Serum antibody complement fixation test for a battery of viral infections was positive only for adenovirus, the titer on admission being $1 / 16,1 / 8$ at 2 weeks, and undetectable at 4 weeks. PCR for adenovirus on lymphocytes and saliva was negative. Brain MRI was normal. Minor unsteadiness, nystagmus, and absent caloric response in the left ear persisted at 1 month. (Zannolli R, Zazzi M, Muraca MC, et al. A child with vestibular neuritis. Is adenovirus implicated? Brain Dev July 2006;28:410-412). (Respond: Dr Raffaella Zannolli: e-mail: zannolli@unisi.it).

COMMENT. This case report is suggestive of an etiologic role for adenovirus infection in vestibular neuritis. Detection of the virus by pharyngeal culture or antigen is the preferred diagnostic method for adenovirus (AAP Red Book, $25^{\text {th }}$ ed, 2000;162-163), and the negative PCR on saliva may militate against a recent infection. A reactivation of a previous 
infection is possible, but biological samples from a previous episode of vertigo were unavailable for testing.

Vestibular neuritis caused by enteroviral infection is reported in a 7-year-old male, and confirmed by isolation of enteroviral RNA in CSF and nasopharyngeal washings. (Ergul Y et al. Pediatr Neurol Jan 2006;34:45-46). Other etiologies listed include mumps, rubella, herpes simplex type 1, cytomegalovirus, and Epstein-Barr virus.

\section{PROGNOSTIC VALUE OF CYTOCHROME C AND CYTOKINES IN ACUTE VIRAL ENCEPHALOPATHY}

Serum cytochrome c and cytokines were evaluated as prognostic predictors in 29 children (ages 9 mos to 9 yrs 11 mos) with viral acute encephalopathies and multiple organ failure at Fukushima Medical University School of Medicine, Japan. Influenza A or AH3 was isolated in 13 patients, HHV-6 in 2, rotavirus (1), enterovirus (1), and the pathogen was unknown in 12. Serum samples collected in the initial phase showed elevated levels of cytochtome c (CTC-c), tumor necrosis factor-a (TNF-a), interleukin 6 (IL-6), soluble TNFreceptor 1 (sTNF-R1), and aspartate aminotransferase (AST), predictive of an unfavorable prognosis. A comparison of day 1 and day 2 measurements showed significant increases in patients who died compared to those who lived. CTC-c was the most sensitive of these markers (93\%), with $100 \%$ specificity, whereas TNF-a had $60 \%$ sensitivity and $100 \%$ specificity. IL-6 was $77 \%$ sensitive and $100 \%$ specific, and AST $82 \%$ and $83 \%$, respectively. These markers are proposed as guides to initial treatment and further intensive care. (Hosoya M, Kawasaki Y, Katayose M et al. Prognostic predictive values of serum cytochrome c, cytokines, and other laboratory measurements in acute encephalopathy with multiple organ failure. Arch Dis Child June 2006;91:469-472). (Respond: Dr M Hosoya, Dept of Pediatrics, Fukushima Medical University School of Medicine, Hikarigaoka-1, Fukushima 960-1295, Japan).

COMMENT. This study confirms previous reports of elevated serum concentrations of pro-inflammatory cytokines, cytokine receptors, and cytochrome $\mathrm{c}$ in children with acute influenza viral encephalopathy, prolonged convulsions, and multiple organ failure with high mortality $(30 \%)$. Brain edema develops on the day after neurologic onset, but fatal outcome is not predicted by neurological symptoms. The authors propose the measurement of cytochrome c and cytokines on day 1 and 2 of the illness as a predictor of prognosis and guide to early intensive treatment.

Surtees $\mathrm{R}$ and DeSousa C, in a perspective on this report (Influenza virus associated encephalopathy. Arch Dis Child June 2006;91:455-456), propose a sequence of events: influenza infection - hypercytokinemia - vascular inflammation - endothelial apoptosis cerebral edema - cerebral necrosis. One third die (multiorgan or brain stem failure), one third survive with sequelae and cerebral atrophy, and one third recover. Reduction of circulating cytokines by plasma exchange or anti-cytokine antibodies might limit vascular damage and prevent encephalopathy.

An elevated CSF/serum-albumin ratio, indicative of an impaired blood-brain barrier, was the earliest sign of acute viral encephalopathy, in a study of 22 patients, the majority having elevated interferon-a levels in CSF and serum (Clarke M et al. Dev Med Child Neurol 2006;48:294-300; Ped Neur Briefs April 2006;20:25-26). 